\title{
Ineficácia da autorregulamentação das propagandas de bebidas alcoólicas: uma revisão sistemática da literatura internacional
}

\author{
Inefficacy of self-regulation of alcohol advertisements: a \\ systematic review of the literature
}

\author{
Alan Vendrame', Ilana Pinsky' \\ ${ }^{1}$ Departamento de Psiquiatria, Universidade Federal de São Paulo (UNIFESP), São Paulo, SP, Brasil
}

\begin{abstract}
Resumo
Objetivo: A literatura científica mais recente aponta que a publicidade de bebidas alcoólicas influencia o comportamento, particularmente o consumo precoce e em grandes quantidades por crianças e adolescentes. Da perspectiva da saúde pública, a publicidade de bebidas alcoólicas deve ser restringida. Em muitos países, assim como no Brasil, os limites são estabelecidos pela autorregulamentação (ou seja, controlados pela própria comunidade publicitária). Analisou-se na presente revisão o conjunto dos trabalhos publicados na literatura internacional sobre este tipo de controle. Método: Realizou-se uma revisão bibliográfica sistemática de trabalhos que investigaram a eficácia da autorregulamentação da publicidade de bebidas alcoólicas. A busca foi feita nas bases de dados Medline, SciELO, Camy e Google Scholar, entre 1991 e 2010, bem como pela técnica de "bola de neve" para a indicação dos principais autores no tema. Foram considerados para o presente propósito 11 artigos. Discussão: $O$ conjunto dos trabalhos obtidos aponta que a autorregulamentação da publicidade de bebidas alcoólicas é pouco eficaz, não cumprindo com os objetivos de evitar, por exemplo, publicidade direcionada às crianças e adolescentes. Conclusão: Outras medidas devem ser consideradas para o controle e a veiculação das propagandas de bebidas alcoólicas, tais como monitoramento independente e controle legal.
\end{abstract}

Descritores: Propaganda; Controle da publicidade de produtos; Consumo de bebidas alcoólicas; Adolescentes; Literatura de revisão como assunto

\section{Abstract}

Objective: The most recent scientific literature indicates that alcohol advertising influences behavior, particularly early and higher alcohol consumption by children and adolescents. From a public health perspective, alcohol advertising should be restricted. In many countries, as well as in Brazil, limits to alcohol advertising are established by industry self-regulation (e.g. controlled by the advertising community itself). We examined in this review all articles on the subject of industry self-regulation of alcohol advertising published in the international literature. Method: A systematic literature review was conducted on articles investigating the effectiveness of self-regulation of alcohol advertisings. The search was conducted in Medline, SciELO, Camy and Google Scholar, between the years of 1991 and 2010. In addition, the "snowball" technique for the indication of the main authors on the subject was employed. From the articles found, 11 focused on the subject discussed here. Discussion: The set of articles obtained indicates that industry self-regulation of alcohol advertising does not show evidence of efficacy. In other words, such a regulation does not prevent, for instance, alcohol advertising directed at children and adolescents. Conclusion: Further measures should be considered for the control and the broadcast of alcohol advertising, such as independent monitoring, legal control.

Descriptors: Propaganda; Products publicity control; Alcohol drinking; Adolescents; Review literature as topic

As estratégias de marketing das indústrias de bebidas alcoólicas tendem a ser globalmente pensadas e aplicadas ${ }^{4,5}$. Isso porque, atualmente, existem grandes conglomerados empresariais multinacionais controlando as principais marcas de bebidas, seja nos mercados desenvolvidos, como naqueles em desenvolvimento ${ }^{4}$. Frequentemente, a marca é associada a esportes, estilos de vida e identidades que variam de acordo com a cultura local ${ }^{6}$. No Brasil, as cervejas são associadas com futebol e carnaval, ao passo que, nos Estados Unidos, com 
eventos esportivos específicos, tais como a liga de baseball e o campeonato nacional de basquete universitário.

Vários estudos conduzidos sob a perspectiva da saúde pública, que analisaram a relação entre a propaganda de bebida alcoólica e o consumo de álcool, mostraram que o conteúdo das mensagens publicitárias atua no processo de tomada de decisão do indivíduo para o consumo de álcool ${ }^{7-12}$. O indivíduo tende a gostar e se identificar com as situações descritas nas propagandas que, de modo recorrente, apelam a temas como humor, erotismo e esportes, frequentemente associando o consumo de álcool com situações agradáveis e engraçadas ${ }^{13-17}$. Desta forma, a resposta afetiva (gostar), a lembrança e a exposição a essas mensagens aumentam a probabilidade de um consumo mais intenso e precoce de bebidas alcoólicas entre os adolescentes ${ }^{18-20}$.

$\mathrm{O}$ discurso dos profissionais de publicidade e da indústria de álcool segue uma linha diferente. Segundo eles, o objetivo da publicidade não é aumentar o consumo, e sim promover a troca e a fidelidade à marca ${ }^{21}$. No entanto, esta afirmação pode suscitar dúvidas em situações específicas. Em relação ao carnaval, por exemplo, a indústria cervejeira admite, em seu relatório anual, a existência de aumento do consumo como resultado de um esforço maior em publicidade ${ }^{22}$. Em relação à questão da fidelidade, estudos já demonstraram que apresentar fidelidade a uma marca, isoladamente, pode predizer consumo em maior quantidade de álcool ${ }^{15-17,23}$. A resposta afetiva positiva que o público de crianças e adolescentes tem em relação às propagandas, associada com o consumo futuro ou atual de álcool ${ }^{7,9,10}$, pode levantar dúvida sobre as afirmações da indústria de que esta não busca aumento do número de consumidores entre os menores de idade ${ }^{24,25}$.

Do ponto de vista da prevenção, estudos econométricos, investigando a disposição da publicidade em determinados mercados, encontraram que a limitação ou o banimento da veiculação da publicidade de bebidas alcoólicas resultou em uma redução do consumo geral de álcool localmente ${ }^{19,26}$. Além disso, adolescentes que viviam em mercados com maior quantidade de publicidade apresentaram maior tendência a consumir mais bebida alcoólica do que aqueles em mercados onde a publicidade era restrita ${ }^{19}$.

Nos países onde a veiculação das propagandas de bebidas alcoólicas é permitida, existem dois modos de regulamentação: através de lei (controle legal) e a autorregulamentação (controle ético), sendo que em alguns casos ambos podem coexistir, um preponderante ao outro ${ }^{27}$.

Por conta de seu caráter multinacional, a indústria do álcool incentiva e defende a autorregulamentação pelo mundo, por meio de códigos de ética próprios ou de órgãos não-governamentais, incluindo publicitários e outros grupos de interesse $e^{21,28-30}$. $\mathrm{Na}$ defesa deste tipo de regulamentação, argumenta-se que a autorregulamentação funciona e é eficaz ao cumprir seus objetivos: proibir mensagens dirigidas a crianças e adolescentes e não incentivar o consumo abusivo e irresponsável de álcool ${ }^{27}$.

O Brasil adotou a autorregulamentação, sob responsabilidade do Conselho Nacional de Autorregulamentação Publicitária
(CONAR). O CONAR é um organismo não-governamental, fundado em 1980, por publicitários que temiam medidas governamentais de proibição da propaganda, que criou um sistema de controle ético da publicidade, por meio da edição de um código de autorregulamentação e de um conselho de ética responsável por julgar eventuais descumprimentos das regras. Para as propagandas de cerveja e vinho, as indústrias seguem a autorregulamentação. Isso porque, apesar da edição da lei 9.294, de 15 de julho de 1996, que regulamenta o horário de veiculação das propagandas de bebidas alcoólicas (livres, das 21 h00 às 6h00), cervejas e vinhos não se enquadram nas restrições legais (a lei considera bebida alcoólica aquela com concentração alcoólica acima de $13^{\circ}$ Gay Lussac), daí porque a autorregulamentação é aplicada para a publicidade dessas bebidas.

As regras e restriçôes contidas no código brasileiro de autorregulamentação publicitária, assim como nos outros países que adotam essa sistemática, decorrem de dois princípios: proteção a crianças e adolescentes, de modo a proibir que as propagandas sejam dirigidas a este público; e a proibição da indução ao consumo abusivo e irresponsável de bebidas alcoólicas. Como decorrência, uma série de limitações é imposta para disciplinar o conteúdo das mensagens publicitárias.

Em razão do recente interesse no tema e da relevância do mesmo para o campo da prevenção de problemas relacionados às bebidas alcoólicas (principalmente entre crianças e adolescentes), o objetivo deste artigo é oferecer uma atualização dos estudos internacionais sobre a eficácia da autorregulamentação da publicidade de bebidas alcoólicas.

\section{Método e Resultados}

Para essa revisão foram utilizados dois métodos: indexadores Medline, SciELO, Camy (banco de dados da área da comunicação) e Google Scholar, entre os anos de 1991 e 2010, sendo que os termos consultados foram "self regulated and alcohol advertising", "ethical control and alcohol advertising". Utilizou-se, ainda, a técnica conhecida como "bola de neve", na qual os autores com produção significativa de artigos sobre autorregulamentação de propaganda de álcool foram contatados para o envio de todos seus trabalhos sobre o assunto, além de sugerirem outros autores que também investigam a questão.

Ao todo, foram obtidos 36 artigos voltados genericamente para o assunto aqui investigado. A maioria dos artigos obtidos não foi considerada porque as expressóes pesquisadas se encontravam ao longo dos artigos, em introdução ou discussão, sem representar o objetivo dos estudos. Portanto, após a exclusão de editoriais e estudos que tratavam de temas tangenciais, como textos opinativos e artigos que traziam as expressões pesquisadas ao longo do texto e não como objetivo, foram selecionados 11 artigos originais, que se encontram listados em duas tabelas. $\mathrm{Na}$ Tabela 1 , os trabalhos foram classificados por ano, país e tipo de publicação; na Tabela 2, por ano de publicação, com o respectivo resumo.

Os trabalhos encontrados são originários de cinco países: Austrália (5), Estados Unidos (2), Itália (2), Reino Unido (1) e 
Tabela 1 - Publicações sobre a autorregulamentação das propagandas de bebidas alcoólicas, sob a perspectiva da saúde pública, desde 1991, organizada por ano e autores

\begin{tabular}{|c|c|c|c|}
\hline Ano & Autores & Título & $\begin{array}{c}\text { País de origem/tipo de } \\
\text { publicação }\end{array}$ \\
\hline $2010^{31}$ & $\begin{array}{l}\text { Vendrame A, Pinsky I, Silva R, } \\
\text { Babor TF }\end{array}$ & $\begin{array}{l}\text { Assessment of self-regulatory code violations in Brazilian } \\
\text { television beer advertisements }\end{array}$ & Brasil / artigo \\
\hline $2010^{32}$ & $\begin{array}{l}\text { Hastings } \mathrm{G} \text {, Brooks } \mathrm{O} \text {, Stead } \\
\mathrm{M}, \text { Angus } \mathrm{K}, \text { Anker } \mathrm{T} \text {, Farrel T }\end{array}$ & Failure of self regulation of UK alcohol advertising & Reino Unido/artigo \\
\hline $2008^{30}$ & Babor TF, Xuan Z, Proctor D & $\begin{array}{l}\text { Reliability of a rating procedure to monitor industry self- } \\
\text { regulation codes governing alcohol advertising content }\end{array}$ & Estados Unidos/artigo \\
\hline $2008^{28}$ & Jones $\mathrm{S}$, Hall $\mathrm{D}$, Munro $\mathrm{G}$ & $\begin{array}{l}\text { How effective is the revised regulatory code for alcohol } \\
\text { advertising in Australia? }\end{array}$ & Australia/artigo \\
\hline $2007^{33}$ & $\begin{array}{l}\text { Donovan } \mathrm{K} \text {, Donavan } \mathrm{R} \text {, Howat } \\
\mathrm{P}, \text { Weller } \mathrm{N} \text {. }\end{array}$ & $\begin{array}{l}\text { Magazine alcohol advertising compliance with the Australian } \\
\text { Alcoholic Beverages Advertising Code }\end{array}$ & Australia/artigo \\
\hline $2007^{29}$ & Jones S, Lynch M. & $\begin{array}{l}\text { Non-advertising alcohol promotions in licensed premises: does } \\
\text { the Code of Practice ensure responsible promotion of alcohol? }\end{array}$ & Australia/artigo \\
\hline $2007^{34}$ & Beccaria F. & Italian alcohol advertising: regulation and enforcement. & Italia/artigo \\
\hline $2005^{35}$ & Zwarun L, Farrar KM & $\begin{array}{l}\text { Doing What They Say, Saying What They Mean: Self-Regulatory } \\
\text { Compliance and Depictions of Drinking in Alcohol Commercials } \\
\text { in Televised Sports }\end{array}$ & Estados Unidos/artigo \\
\hline $2002^{36}$ & Jones S, Donovan R. & Self-regulation of alcohol advertising: Is it working for Australia & Australia/artigo \\
\hline $2001^{37}$ & Beccaria, $F$. & The Italian debate on alcohol advertising regulation. & Italia/artigo \\
\hline $1991^{38}$ & Saunders B, Yap E. & $\begin{array}{l}\text { Do our guardians need guarding? An examination of the } \\
\text { Australian system of self-regulation of alcohol advertising }\end{array}$ & Australia/artigo \\
\hline
\end{tabular}

Brasil (1). O interesse no tema é recente, o que explica o fato de que oito deles foram publicados entre os anos de 2007 e 2010.

Pesquisadores australianos estudam o tema desde o início da década de 1990. Em geral, o foco dos trabalhos foi analisar a eficácia da autorregulamentação, pela comparação do julgamento de denúncias contra propagandas de álcool apresentadas pelo público geral ao órgão responsável pelo controle ético, com o julgamento de experts e de pessoas do público geral.

No caso dos pesquisadores americanos, observa-se que as publicaçôes se dividem de acordo com os objetos. O primeiro artigo teve como objeto a análise das propagandas veiculadas em eventos esportivos. Especificamente, esse artigo visava verificar se em tais ocasiōes as normas do código de autorregulamentação eram devidamente seguidas.

O segundo artigo descreve um trabalho metodológico sofisticado, desenvolvido pelo pesquisador Thomas Babor que, desde o início de 2000, dedica-se a construir um procedimento confiável de monitoramento independente das propagandas de bebidas alcoólicas, tanto por experts, como por avaliadores não treinados.

Os artigos italianos descrevem estudos preliminares sobre o sistema de autorregulamentação para as propagandas de bebidas alcoólicas. Assim como nos casos australiano e americano, a autorregulamentação seria falha na Itália, não sendo suficiente para limitar os conteúdos das propagandas italianas.

$\mathrm{O}$ artigo do Reino Unido, bastante recente, remete a um estudo realizado por um comitê de saúde, a pedido da Câmara dos Comuns, para analisar a efetividade e o sucesso da autorregulamentação para as propagandas de bebidas alcoólicas. O comitê obteve grande número de documentos internos das indústrias do álcool e suas agências de publicidade, a fim de examinar o pensamento e o planejamento estratégico em que se baseia a promoção de álcool, para saber não só aquilo que os anunciantes estão dizendo, mas porque eles estão dizendo o que é veiculado nas peças publicitárias.

O estudo revelou que, apesar de conhecerem as proibições e limitações impostas pela autorregulamentação, tais como evitar mensagens dirigidas a menores de 18 anos, bem como atores e personagens menores de 25 anos de idade, as regras foram sistematicamente violadas, ainda no planejamento das campanhas. Documentos revelaram que pesquisas qualitativas com adolescentes entre 14 e 17 anos foram utilizadas para estruturar determinadas campanhas de cerveja. O estudo mostrou que as indústrias e os publicitários não levaram em consideração as limitações e restrições impostas pelas regras da autorregulamentação, pensando no marketing do álcool como se fosse um produto qualquer.

No Brasil, o tema ganhou interesse com a formação, em 2004, de um grupo de pesquisa (do qual os dois autores desse artigo fazem parte) com apoio da Fundação de Amparo à Pesquisa do Estado de São Paulo (FAPESP), que estuda as propagandas de bebidas alcoólicas do ponto de vista da saúde pública. $\mathrm{O}$ artigo brasileiro analisou a eficácia das normas da autorregulamentação, a partir do julgamento do público adolescente que, em princípio, deveria ser protegido do conteúdo das mensagens. Este tipo de análise, a partir do ponto de vista de uma população vulnerável ao conteúdo das mensagens, até então não tinha sido realizada. Ao 


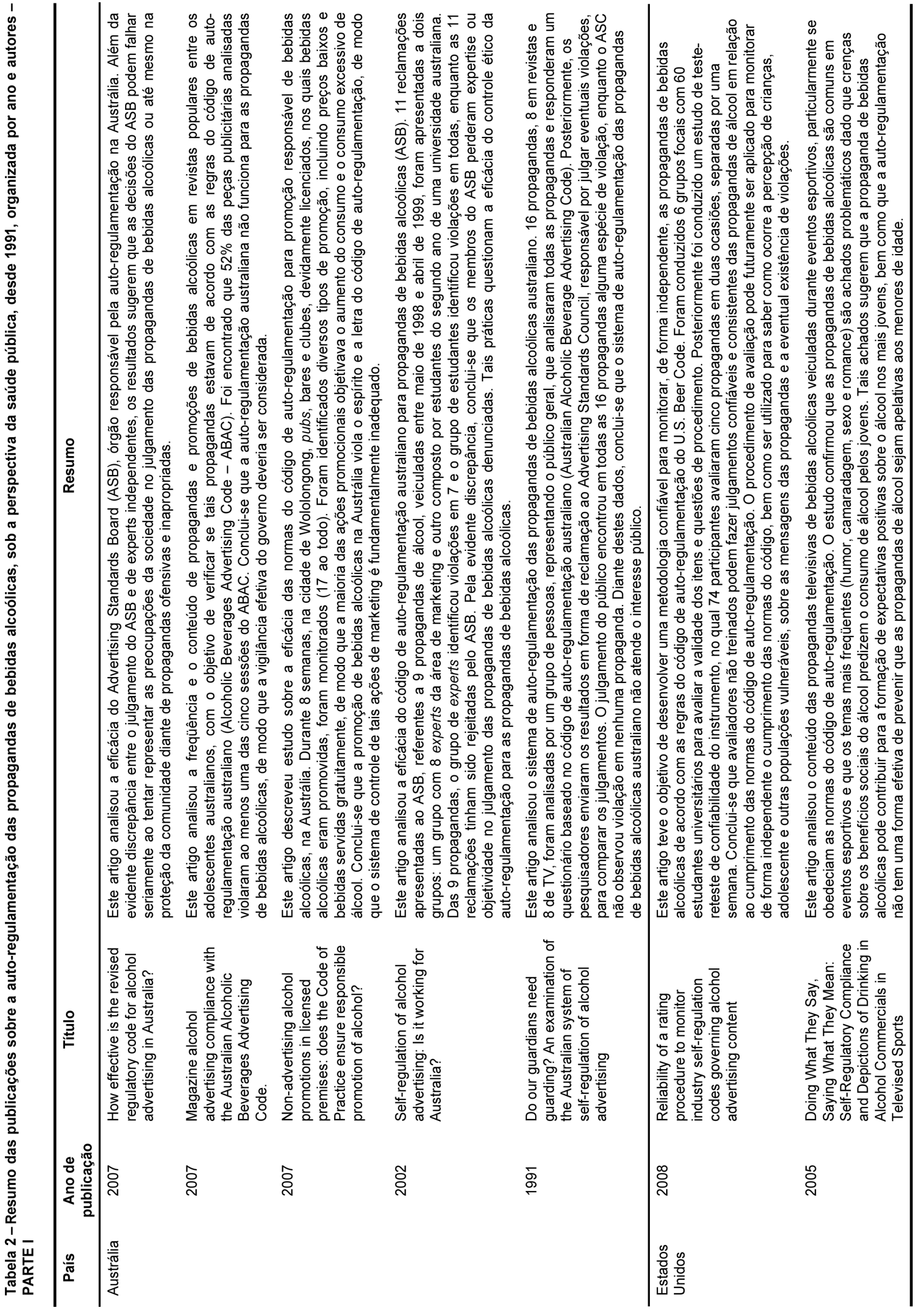



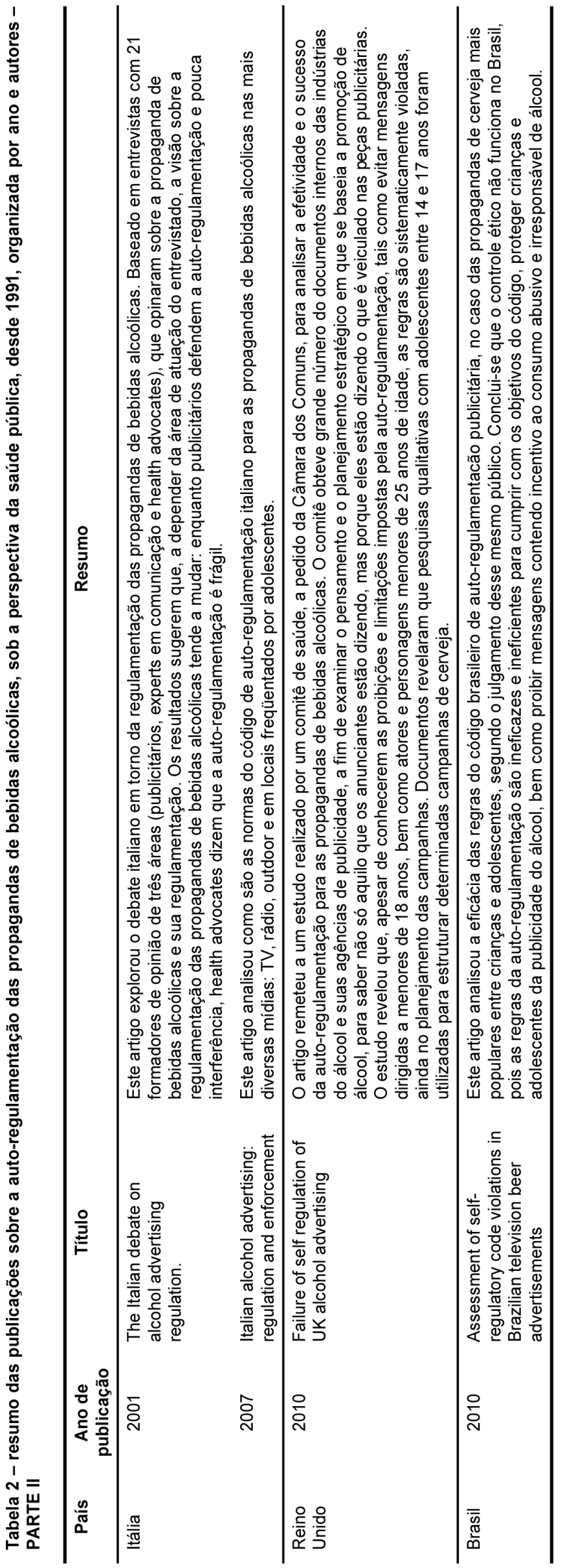

mesmo tempo, buscou-se o desenvolvimento de uma metodologia capaz de proporcionar condições de se criar um monitoramento independente das propagandas de bebidas alcoólicas veiculadas na televisão, especificamente se elas se enquadram nas regras da autorregulamentação.

Além deste aspecto metodológico inovador, os pesquisadores constataram uma série de violações às regras do código brasileiro de autorregulamentação publicitária. Todas as propagandas avaliadas (cinco propagandas das marcas mais populares entre os adolescentes) violaram significativamente as regras do código. No total, das 15 regras avaliadas, 11 foram violadas.

\section{Discussão}

O presente artigo teve o objetivo de revisar sistematicamente a literatura dos estudos sobre a autorregulamentação das propagandas de bebidas alcoólicas. Estudos originários de cinco diferentes países apontaram que o controle do conteúdo e da frequência da publicidade de álcool pelo próprio grupo de produtores da publicidade dá margem a interpretações subjetivas e falha em seu objetivo de proteger públicos vulneráveis, bem como de evitar mensagens que incentivem o consumo irresponsável e exagerado de álcool.

Atualmente, existe um conhecimento bem estabelecido na literatura apontando que a publicidade de álcool atinge e influencia o público de crianças e adolescentes quanto ao consumo de álcool ${ }^{23-25,39,40}$ e que, por tais razôes, pela perspectiva da saúde pública, as propagandas de bebidas alcoólicas devem ser limitadas.

Do ponto de vista da prevenção dos problemas relacionados ao álcool, a ciência tem papel relevante, na medida em que, ao produzir o conhecimento de fatores de risco, indica caminhos para a criação de políticas públicas baseadas em evidências. $\mathrm{O}$ que se percebe dos trabalhos reunidos neste artigo é que o controle e a regulamentação das propagandas de bebidas alcoólicas ainda estão longe de cumprir o objetivo de evitar mensagens dirigidas a crianças e adolescentes. Os resultados mostraram que o julgamento das regras, por adolescentes e experts, apontou violaçôes, enquanto a avaliação dos órgãos responsáveis pelo controle ético tendeu a não concluir pela existência delas. Portanto, não só as regras dos códigos de autorregulamentação, como também o sistema de controle das propagandas pode ser questionado como princípio.

Os estudos australianos mostraram que o órgão responsável pelo controle das propagandas de bebidas alcoólicas é falho ao avaliar denúncias de propagandas em desacordo com as normas do código de autorregulamentação. Enquanto o órgão não encontrava violaçóes, avaliações de experts e do público geral perceberam o descumprimento do que ordenava o código ${ }^{28,36}$. Isso pode ser explicado pela complexidade e detalhamento das normas, o que permite avaliaçóes preponderantemente subjetivas, mesmo em desarmonia com o senso comum.

Assim como na Austrália, nos Estados Unidos, Brasil e Itália o cenário é semelhante: regras complexas, abertura para julgamentos subjetivos e pouco controle do que é veiculado nas propagandas de bebidas alcoólicas. Os estudos americanos observaram que, 
sob avaliação de experts ou pessoas não-treinadas (como é o público geral), as propagandas de bebidas alcoólicas violam significativamente o código de autorregulamentação ${ }^{29,35}$. Do mesmo modo, no Brasil, quando a população que deveria ser protegida avalia as propagandas, violações significativas são encontradas, o que torna a situação preocupante, pelo fato de que a população vulnerável é um dos alvos diretos das mensagens, sem qualquer tipo de proteção eficaz ${ }^{31}$.

\section{Conclusão}

Interessante notar que as falhas do sistema da autorregulamentação das propagandas de bebidas alcoólicas são semelhantes no mundo todo, independentemente de características culturais, sociais ou econômicas. Aparentemente de forma sistemática, a autorregulamentação não evita o direcionamento das propagandas às crianças e adolescentes, e tão pouco evita mensagens de incentivo ao consumo abusivo e irresponsável de álcool. Parece que as indústrias e as agências de publicidade, cientes da complexidade das regras e das falhas no controle, pensam e concretizam estratégias publicitárias para atender os próprios interesses, tais como aumentar o volume de vendas e conquistar novos consumidores, ainda que menores de idade. $\mathrm{O}$ estudo do Reino Unido é importante neste sentido, pois revelou como a indústria do álcool e os publicitários pensam e constroem as campanhas publicitárias. Tal como foi observado, as estratégias levam em conta o aumento das vendas e a conquista de novos consumidores, mesmo que sejam dirigidas a menores de 18 anos de idade e que incentivem o consumo abusivo de álcool.

Tais constatações podem servir de fundamento para a criação de políticas públicas, no sentido de restringir e regulamentar a veiculação e o conteúdo das propagandas de bebidas alcoólicas. $\mathrm{O}$ argumento das indústrias de bebidas e dos setores da publicidade e propaganda de que os anúncios não se direcionam ao público adolescente menor de idade, porque visam única e exclusivamente à fidelidade à marca ${ }^{21}$, pode ser questionado diante dos dados reunidos. A questão talvez seja que as estratégias de marketing das indústrias do álcool têm o objetivo de promover a bebida como se fosse um produto qualquer, atingindo, inclusive, o público de crianças e adolescentes.

Medidas como a criação de um corpo independente de monitoramento das propagandas, sem qualquer vínculo com a indústria, seria uma alternativa a considerar. Os dados avaliados mostraram que quando as propagandas foram analisadas por grupos externos, sem relação de interesse com o produto ou a publicidade (por grupo de experts ou por adolescentes), em diferentes países, um número considerável de violações do código de autorregulamentação foi revelado.

\section{Agradecimentos}

À Fundação de Amparo à Pesquisa do Estado de São Paulo (FAPESP), financiadora do projeto de pesquisa que deu origem ao presente artigo (2003/06250-7 e 04/13564-0).

Financiamento e conflito de interesses

\begin{tabular}{|c|c|c|c|c|c|c|c|}
\hline $\begin{array}{c}\text { Membro do } \\
\text { grupo de autores }\end{array}$ & Local de trabalho & $\begin{array}{l}\text { Verba de } \\
\text { pesquisa }^{1}\end{array}$ & $\begin{array}{c}\text { Outro apoio à } \\
\text { pesquisa ou educação } \\
\text { médica continuada }\end{array}$ & $\begin{array}{c}\text { Honorários } \\
\text { de } \\
\text { palestrantes }\end{array}$ & $\begin{array}{l}\text { Participação } \\
\text { acionária }\end{array}$ & $\begin{array}{l}\text { Consultor/ } \\
\text { conselho } \\
\text { consultivo }\end{array}$ & Outro $^{3}$ \\
\hline Ilana Pinsky & UNIFESP/EPM & FAPESP ${ }^{* *}$ & - & - & - & - & - \\
\hline $\begin{array}{l}\text { * Modesto } \\
\text { ** Significativa } \\
{ }^{* *} \text { Significativa. Mor } \\
\text { Nota: UNIFESP/EP } \\
\text { Estado de São Paul } \\
\text { Mais informações, C }\end{array}$ & $\begin{array}{l}\text { tes fornecidos à ir } \\
\text { Escola Paulista }\end{array}$ & $\begin{array}{l}\text { tuição do at } \\
\text { Medicina, } \\
\text { s Autores. }\end{array}$ & $\begin{array}{l}\text { ou a colega onde o auto } \\
\text { versidade Federal de S }\end{array}$ & $\begin{array}{l}n \text { participaçã } \\
\text { Daulo: FAPE }\end{array}$ & $\begin{array}{l}\text { o diretamen } \\
\text { Fundação }\end{array}$ & autor. & is \\
\hline
\end{tabular}

\section{Referências}

1. Laranjeira R, Pinsky I, Zaleski M, Caetano R. I Levantamento Nacional sobre os padrões de consumo de álcool na população brasileira. Secretaria Nacional Anti-Drogas; 2007.

2. Galduróz JC, Caetano R. Epidemiology of alcohol use in Brazil. Rev Bras Psiquiatr. 2004;26(Suppl 1):S3-6.

3. Pinsky I, Zaleski M, Caetano R, Sanches M, Laranjeira R. Alcohol use patterns among Brazilian adolescents. Rev Bras Psiquiatr. 2010;32(3):242-9.

4. Jernigan D. The global expansion of alcohol marketing: ilustrative case studies and recommendations for action. J Public Health Policy. 1999;20(1):56-80.

5. Jernigan, D. The global alcohol industry: an overview. Addiction. 2009;104(Supppl. 1):6-12.

6. Pinsky I, Silva M. A frequency and content analisys of alcohol advertising on brasilian television. J Stud Alcohol. 1999;60(3):394-9.

7. Casswell S, Zhang JF. Impact of liking for advertising and brand allegiance on drinking and alcohol-related aggression: a longitudinal study. Addiction. 1998;93(8):1209-17.
8. Jernigan DH, Ostroff J, Ross C. Alcohol advertising and youth: a measured approach. J Public Health Policy. 2005;26(3):312-25.

9. Austin EW, Hust SJ. Targeting adolescents? The content and frequency of alcoholic and nonalcoholic beverage ads in magazine and video formats: November 1999-April 2000. J Health Commun. 2005;10(8):769-85.

10. Collins RL, Ellickson PL, McCaffrey D, Hambarsoomians K. Early adolescent exposure to alcohol advertising and its relationship to underage drinking. $J$ Adoles Health. 2007;40(6):527-34.

11. Austin EW, Chen MJ, Grube JW. How does alcohol advertising influence underage drinking? The role of desirability, identification and skepticism. J Adolesc Health. 2006;38(4):376-84.

12. Babor T, Caetano R, Casswell S, Edwards G, Giesbrecht N, Graham E, Rehm J, Room R, Rossow I. Alcohol: no ordinary commodity. Oxford Medical Publications; 2003.

13. Fleming K, Thorson E, Atkin CK. Alcohol advertising exposure and perceptions: links with alcohol expectancies and intentions to drink or drinking in underaged youth and young adults. J Health Commun. 2004;9(1):3-29. 
14. Stacy AW, Zogg JB, Unger JB, Dent CW. Exposure to televised alcohol ads and subsequent adolescent alcohol use. Am J Health Behav. 2004;28(6):498509.

15.Collins RL, Schell T, Ellickson PL, McCaffrey D. Predictors of beer advertising awareness among eighth graders. Addiction. 2003;98(9):1297-306.

16. Wyllie A, Zhang JF, Casswell S. Responses to televised alcohol advertisements associated with drinking behavior of 10-17-year-olds. Addiction. 1998;93(3):361-71.

17. Grube JW, Wallack L. Television beer advertising and drinking knowledge, beliefs, and intentions among schoolchildren. Am J Public Health. 1994;84(2):254-9.

18. Connolly GM, Casswell S, Zhang JF, Silva PA. Alcohol in the mass media and drinking by adolescents: a longitudinal study. Addiction. 1994;89(10):1255-63.

19. Snyder LB, Milici FF, Slater M, Sun H, Strizhakova Y. Effects of alcohol advertising exposure on drinking among youth. Arch Pediatr Adolesc Med. 2006;160(1):18-24.

20. Hastings G, Anderson S, Cooke E, Gordon R. Alcohol marketing and young people's drinking: a review of the research. I Public Health Policy. 2005;26(3):292-5.

21. Narchi, E. O álcool nos meios de comunicação. Audiência Pública realizada pelo Conselho de Comunicação Social do Congresso Nacional. In: Exposição do CONAR. Brasília, maio 2005. [citado 12 out 2010]. Available from http://www.senado.gov.br 22. Grupo Schincariol. Marketing e Produto. Relatório Anual 2006. São Paulo; 2006. p.22.

23. Casswell S, Zhang JF. Impact of liking for advertising and brand allegiance on drinking and alcohol-related aggression: a longitudinal study. Addiction. 1998;93(8):1209-17.

24. Vendrame A, Pinsky I, Faria R, Silva R. Brazilian teenagers and beer advertising: relationship between exposure, positive response, and alcohol consumption. Cad Saude Publica. 2009; 25(2):359-65.

25. Ellickson PL, Collins RL, Hambarsoomians K, McCaffrey DF. Does alcohol advertising promote adolescent drinking? Results from a longitudinal assessment. Addiction. 2005;100(2):235-46.

26. Saffer $\mathrm{H}$. Alcohol consumption and alcohol advertising bans. NBER Work Paper Series - work paper 7758; 2000 (http://papers.nber.org/papers/w7758).

27. Pinsky I (coord), Vendrame A, El Jundi, S. Publicidade de bebidas alcoólicas e os jovens. Fapesp; 2009.

28. Jones S, Hall D, Munro G. How effective is the revised regulatory code for alcohol advertising in Australia? Drug Alcohol Rev. 2008;27(1):29-38.

29. Jones S, Lynch M. Non-advertising alcohol promotions in licensed premises: does the Code of Practice ensure responsible promotion of alcohol? Drug Alcohol Rev. 2007;26(5)477-85.

30. Babor TF, Xuan Z, Proctor D. Reliability of a rating procedure to monitor industry self-regulation codes governing alcohol advertising content. J Stud Alcohol Drugs. 2008;69(2):235-42.

31. Vendrame A, Pinsky I, Silva RS, Babor T. Assessment of self-regulatory code violations in Brazilian television beer advertisements. J Studies Alcohol Drugs. 2010;71(3):445-51.

32. Hastings G, Brooks O, Stead M, Angus K, Anker T, Farrel T. Failure of self regulation of UK alcohol advertising. BMJ. 2010;340:b5650.

33. Donovan K, Donavan R, Howat P, Weller N. Magazine alcohol advertising compliance with the Australian Alcoholic Beverages Advertising Code. Drug Alcohol Rev. 2007;26(1)73-81.

34. Beccaria F. Italian alcohol advertising: regulation and enforcement. Contemp Drug Probl. 2007;34(1):25-52.

35. Zwarun L, Farrar KM. Doing What They Say, Saying What They Mean: SelfRegulatory Compliance and Depictions of Drinking in Alcohol Commercials in Televised Sports. Mass Comm Soc. 2005;8(4)347-71.

36. Jones $S$, Donovan R. Self-regulation of alcohol advertising: Is it working for Australia? J Public Affairs. 2002;2(3)153-65(13).

37. Beccaria F. The Italian debate on alcohol advertising regulation. Contemp Drug Probl.. 2001;28:719.

38. Saunders B, Yap E. Do our guardians need guarding? An examination of the Australian system of self-regulation of alcohol advertising. Drug Alcohol Rev. $1991 ; 10(1) 15-27$.
39. Anderson P, de Bruijn A, Angus K, Gordon R, Hastings G. Impact of alcohol advertising and media exposure on adolescent alcohol use: a systematic review of longitudinal studies. Alcohol Alcohol. 2009;44(3):229-43.

40. Smith LA, Foxcroft DR. The effect of alcohol advertising, marketing and portrayal on drinking behaviour in young people: systematic review of prospective cohort studies. BMC Public Health. 2009;9:51. 